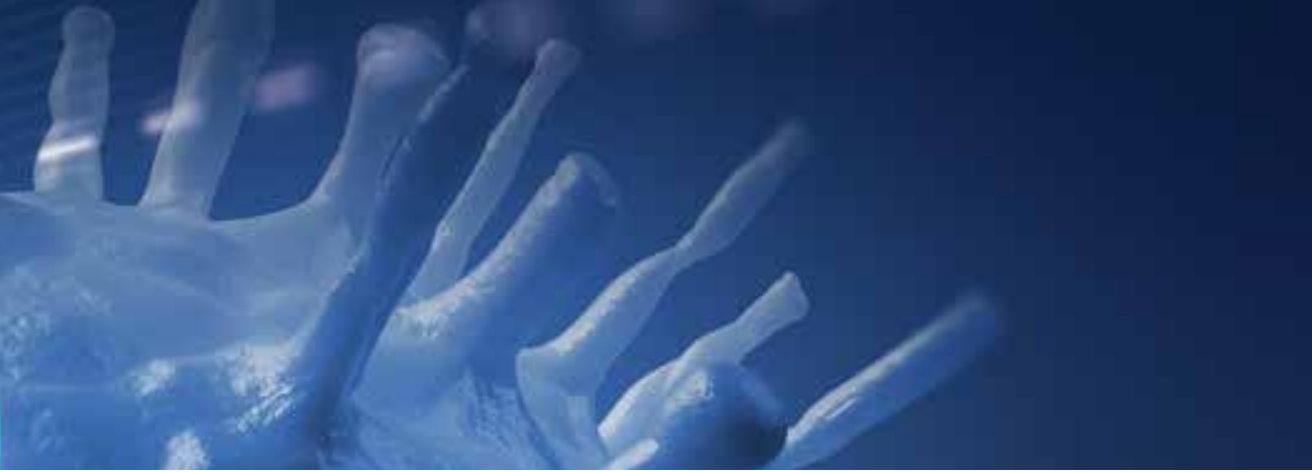

\title{
Violencia contra niñas, niños y adolescentes en tiempos de COVID-19'
}

CEPAL - UNICEF-Oficina de la

Representante Especial del Secretario General sobre la Violencia contra los Niños

- La negligencia y la violencia física, sexual y psicológica, las prácticas nocivas como el matrimonio infantil y las uniones tempranas son formas en que niñas, niños y adolescentes (toda persona menor de 18 años) ven vulnerados su integridad y sus derechos (UNICEF, 2015). Durante décadas se ha advertido que ningún país es inmune a este flagelo, que las diversas manifestaciones de la violencia pueden presentarse en el hogar, en la escuela, en las instituciones de cuidado o de justicia, en la comunidad o en los entornos digitales (Pinheiro, 2006) y que el 50\% de la población de menos de 18 años ha sido víctima de violencia en el hogar, en su mayoría perpetrada por familiares o personas cercanas (OMS, 2020a). Esta violencia afecta de manera diferente a niñas y niños y puede darse en contextos donde existen factores adicionales de riesgo que dan lugar a otras formas de violencia. Entre estos se incluyen las violaciones a los derechos de la niñez y adolescencia en el conflicto armado (Naciones Unidas, 2013), la trata de niñas, niños y adolescentes (UNODC, 2020) y el trabajo infantil (OIT, 1999), que afectan la integridad física, psicológica y social de la niñez y adolescencia y limitan sus posibilidades de desarrollo.

- Durante la crisis de la enfermedad por coronavirus (COVID-19), factores como las limitaciones de la actividad económica, el cierre de las escuelas, el acceso reducido a los servicios de salud y el distanciamiento físico pueden incrementar la vulnerabilidad y exposición en la infancia y adolescencia a la violencia y otras vulneraciones a los derechos de niñas, niños y adolescentes (Bhatia y otros, 2020; UNICEF, 2020b). En América Latina y el Caribe, el deterioro progresivo de los factores socioeconómicos en la última década (CEPAL, 2020a) ha reducido elementos esenciales de protección y puede generar un incremento aún más marcado de la

\section{Índice}

I. ¿Cuáles son las formas de violencia en el hogar? 2

II. Desequilibrio entre los factores de riesgo y los factores de protección en

América Latina y el Caribe 4

III. Los riesgos exacerbados durante la crisis del COVID-19.

IV. La protección de la niñez y adolescencia como prioridad de la nueva agenda.......... 8

Bibliografía .......................................................... 13

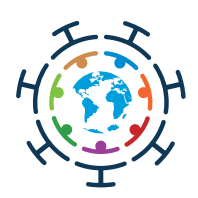


violencia contra niñas, niños y adolescentes en tiempos de COVID-19 que antes de la crisis (OMS, 2020a). Factores como las desigualdades preexistentes en aspectos socioeconómicos, de vivienda y de acceso a la salud hacen que ciertos grupos, como las familias migrantes, se enfrenten a un mayor riesgo de contraer el virus y de sufrir las consecuencias derivadas del impacto de la crisis en los países de la región (Tres y Chatruc, 2020).

- El desequilibrio entre los factores de riesgo y los factores de protección (UNICEF, 2020a) se produce en la medida en que los países imponen normas de distanciamiento físico para ayudar a contener la pandemia (OMS, 2020b). Dada la desigualdad socioeconómica de la región, no todas las personas logran cumplir las medidas sanitarias con igual rigor (CEPAL, 2020b), lo que supone un mayor riesgo para niñas, niños y adolescentes, especialmente aquellos que se encuentran en situaciones en las que son más vulnerables ${ }^{2}$ a ser víctimas de la trata de personas o la explotación sexual (UNODC, 2020), el trabajo infantil (OIT/CEPAL, 2020) o delitos sexuales, incluso a través de medios digitales (INTERPOL, 2020; EUROPOL, 2020). En el contexto de la pandemia de COVID-19, el hogar es donde más tiempo pasan muchas niñas, niños y adolescentes, con sus padres, madres y cuidadores (UNICEF, 2020b), y el entorno digital es el que posibilita que puedan mantener la interacción con otros entornos, como la escuela y la comunidad (INTERPOL, 2020). Dentro del hogar y la familia ${ }^{3}$ es, no obstante, donde se perpetra con mayor frecuencia violencia contra niñas, niños y adolescentes, así como contra mujeres adultas. Si bien el impacto de la violencia puede variar cuando se perpetra contra personas adultas, ambos colectivos sufren consecuencias similares, como los efectos en la salud mental y en la salud sexual y reproductiva, y comparten factores de riesgo, como las normas sociales que toleran ambos tipos de violencia y desalientan la búsqueda de ayuda (Guedes y otros, 2016). Esto resulta relevante, ya que la violencia presenciada por niñas, niños o adolescentes los impacta física y psicológicamente de formas muy similares a cuando son víctimas directas (UNICEF, 2006).

- En el presente documento se abordan el agravamiento de los riesgos y la disminución de los factores de protección ante la violencia física, psicológica y sexual a la que se exponen en su hogar los adolescentes, niños y —especialmente-niñas de la región durante la pandemia de COVID-19. Asimismo, se ofrecen recomendaciones sobre cómo integrar acciones concretas en los mecanismos de respuesta que los Estados de América Latina y el Caribe han dispuesto para enfrentar la crisis del COVID-19.

\section{I. ¿Cuáles son las formas de violencia en el hogar?}

- La violencia contra niñas, niños y adolescentes no es algo que les suceda a las víctimas de manera fortuita. La violencia es un acto deliberado que comete una tercera persona $y$, en esa medida, se debe y se puede prevenir. En el hogar pueden darse condiciones que aumenten la posibilidad de violencia contra las niñas, los niños y los adolescentes por parte de sus padres, madres, cuidadores, pares, hermanas y hermanos, 0 familiares y personas cercanas con quienes interactúan con regularidad o conviven (Alianza para la Protección de la Infancia en la Acción Humanitaria, 2020). Esos son los factores que se debe identificar y atender oportunamente para evitar que se produzca una situación de violencia. Sin embargo, la violencia de la que son víctimas niñas, niños y adolescentes en el hogar no se limita únicamente a la infligida por miembros del hogar o personas cercanas. El incremento del uso de herramientas digitales como
El Comité de los Derechos del Niño define el castigo físico y humillante como aquel en el que "(...) se utilice la fuerza física y que tenga por objeto causar cierto grado de dolor o malestar, aunque sea leve. (...) Además hay otras formas de castigo que no son físicas, pero que son igualmente crueles y degradantes y, por lo tanto, incompatibles con la Convención [sobre los Derechos del Niño]. Entre estas se cuentan, por ejemplo, los castigos en que se menosprecia, se humilla, se denigra, se convierte en chivo expiatorio, se amenaza, se asusta 0 se ridiculiza al niño

Observación General № 8, artículo 11 (Naciones Unidas, 2006)

Niñas, niños y adolescentes refugiados, apátridas, migrantes, privados de libertad, internados, o que ya son víctimas de vulneraciones como trabajo infantil o que se encuentran en situaciones de conflicto o fragilidad (UNICEF, 2020e).

En el presente documento se considera la amplia diversidad de hogares existentes en la región, entre los que se incluyen configuraciones de hogares monoparentales, con mujeres u hombres cabeza de hogar; hogares biparentales tradicionales; convivencia con padrastros o madrastras; hogares extendidos, o jefaturas de hogar en cabeza de personas mayores, con ausencia de padres y madres. 
consecuencia de las medidas de aislamiento adoptadas en la mayoría de los países en el marco de la pandemia (Blackman y otros, 2020) amplía el espectro de potenciales agresores fuera del núcleo familiar con quienes pueden interactuar desde el entorno digital, como pares o personas desconocidas (UIT, 2020; EUROPOL, 2020).

\section{A. Violencia física y psicológica expresada a través del castigo físico y humillante y tratos degradantes}

- El castigo físico es muy común incluso durante los primeros cinco años de vida de los niños y niñas (UNICEF, 2020a) y se relaciona con la violencia psicológica como práctica de crianza (Naciones Unidas, 2006). En estimaciones recientes se calcula una prevalencia de un $55,2 \%$ de la agresión física y un $48 \%$ de la agresión psicológica en la crianza en América Latina y el Caribe (Cuartas, 2019).

\section{B. Violencia sexual}

- La violencia sexual se considera una grave violación de los derechos de niñas, niños y adolescentes y puede tomar la forma de abuso, acoso o explotación sexual (UNICEF, s/fb). Se trata de una actividad sexual influida por el desequilibrio en la relación de poder. En el caso de la explotación sexual, media la retribución o promesa de retribución directa o a una tercera persona (ECPAT Internacional, 2020a). En

La violencia sexual en línea incluye (...) la seducción [grooming], la transmisión en vivo [streaming], el consumo de materiales de abuso sexual infantil y la coerción o chantaje a niñas, niños y adolescentes con fines sexuales.

(ECPAT International, 2018) relación con los casos de violencia sexual ocurrida en el hogar, llama la atención que la mayoría de los actos reportados hasta antes de la pandemia habían sido perpetrados por parte de familiares o personas cercanas (OMS, 2020a), y las niñas y las adolescentes constituyeron la mayoría de las víctimas.

\section{Violencia en el entorno digital}

- Las tecnologías de la información y las comunicaciones (TIC) están siendo uno de los medios más importantes durante la crisis del COVID-19. Si bien existe una deuda en materia de cobertura y penetración de Internet en los países de la región, el mundo digital es el mejor puente para la continuidad de actividades cotidianas como la educación, el trabajo y la comunicación (Trucco y Palma, 2020). Sin embargo, una mayor exposición al entorno digital puede a su vez elevar la exposición a riesgos inherentes a las TIC, tales como el ciberacoso o la violencia sexual por medios digitales, de los que pueden ser víctimas niñas, niños y adolescentes (EUROPOL, 2020). El ciberacoso consiste en la intimidación ${ }^{4}$ repetitiva por medio de las redes sociales, plataformas de mensajería, comunidades virtuales, juegos o teléfonos celulares que busca atemorizar, enfadar o humillar a otras personas (UNICEF, s/fa). El ciberacoso está relacionado con la intimidación sistemática en entornos diferentes al digital, y el aumento de las horas que pasan en Internet niñas, niños y adolescentes eleva significativamente el riesgo de que ocurra (UIT, 2020).

- Es importante tener presente que la violencia en línea no está separada de la que se produce en entornos reales (OMS, 2020a) y que, en el caso de la violencia sexual en línea, puede afectar también a niñas, niños y adolescentes que ni siquiera tienen acceso a un dispositivo electrónico. Hoy en día es común que personas adultas tengan acceso a cámaras digitales, portátiles o celulares que facilitan el registro de actividad sexual con niñas, niños y adolescentes, así como su distribución a través de canales digitales desde sus propios hogares (ECPAT Internacional, 2020b).

\section{Relación entre la violencia contra las mujeres y la violencia contra niñas, niños y adolescentes}

- La violencia contra las mujeres y la violencia contra niñas, niños y adolescentes comparten factores de riesgo, y se ven afectadas por normas sociales similares que conducen a la perpetración y tolerancia de estos fenómenos. Con frecuencia, dichas normas sociales desalientan la búsqueda

4 A través de la difamación o la divulgación de material audiovisual vergonzoso para la víctima o mensajes hirientes o amenazantes, o de la suplantación (UNICEF, s/fa). 
de ayuda y permean los episodios de violencia dentro del hogar o en la propia familia (Guedes y otros, 2016). Estas normas sociales están marcadas por concepciones patriarcales sobre los roles de género, que tienen además repercusiones negativas en los roles y las prácticas de crianza. Por otro lado, la exposición de niñas, niños y adolescentes a la violencia perpetrada hacia otras personas tiene efectos físicos y psicológicos similares a los de la violencia experimentada directamente (UNICEF, 2006, CEPAL/UNICEF, 2018). Adicionalmente, la violencia sistemática contra otros miembros del hogar es en sí misma un factor de riesgo significativo de violencia hacia este grupo etario.

- Una revisión exhaustiva reciente de las investigaciones sobre la violencia contra la mujer y la violencia contra niñas, niños y adolescentes en América Latina y el Caribe (Bott y Ruiz-Celis, 2019) permitió ratificar los hallazgos de la Organización Panamericana de la Salud en 2016 (Guedes y otros, 2016) sobre las intersecciones entre la violencia perpetrada contra las mujeres y la violencia contra niñas, niños y adolescentes. El estudio concluyó que, en la región, estas intersecciones se manifiestan en aspectos como las normas sociales que promueven y justifican la violencia física como método de disciplina en la crianza y en la relación de pareja; en 2019 en la región hasta el $36 \%$ de las mujeres estimaba necesario el uso del castigo físico en la crianza y hasta el 16,6\% consideraba justificable que el esposo golpeara a la esposa (Bott y Ruiz-Celis, 2019).

\section{E. Los efectos de la violencia}

- Durante décadas se ha advertido sobre la violencia contra niñas, niños y adolescentes, que se da en todos los países del mundo, y sus efectos físicos y psicosociales a corto, mediano y largo plazo en las víctimas y en la sociedad (Pinheiro, 2006). La exposición a la violencia, particularmente en los primeros años de vida, afecta la estructura del cerebro y genera un deterioro permanente de las capacidades cognitivas y emocionales, además de predisponer a conductas de alto riesgo y comportamientos antisociales (Cuartas, 2019). Esto significa que la vida de niñas, niños y adolescentes que son víctimas de violencia puede verse impactada en varias dimensiones: menor rendimiento escolar, menores habilidades para relacionarse con los demás y para establecer vínculos afectivos saludables, mayor predisposición a conductas sexuales irresponsables o al uso indebido de sustancias psicoactivas y predisposición a trastornos crónicos de salud mental (Cuartas, 2019).

\section{Desequilibrio entre los factores de riesgo y los factores}

\section{de protección en América Latina y el Caribe}

- Si bien se produce violencia en todos los grupos socioeconómicos, las condiciones socioeconómicas precarias se asocian a una mayor exposición a riesgos y a la reducción de los factores de protección. Elementos de protección ajenos al individuo, como el acceso adecuado a las tecnologías y la información, la seguridad y estabilidad económicas, la educación y el conocimiento sobre la niñez, el cuidado y la crianza son más escasos entre las personas en situación de pobreza, quienes, a su vez, están más expuestas a personas y entornos violentos, el uso problemático de sustancias psicoactivas y la naturalización de las conductas violentas (UNICEF, 2020a). Adicionalmente, cuando la crisis del COVID-19 alcanzó a América Latina y el Caribe la región ya estaba sumida en una crisis migratoria, principalmente derivada del éxodo desde la República Bolivariana de Venezuela hacia otros países de la región y desde Centroamérica hacia América del Norte (Tres y Chatruc, 2020), que se ha traducido en limitaciones en el acceso a la vivienda, a los servicios básicos de agua y saneamiento y a la salud, entre otros. La población migrante encuentra dificultades para acceder a derechos y servicios básicos, por lo que está expuesta a más riesgos y cuenta con menos de los ya limitados factores de protección (Naciones Unidas, 2020b). La crisis del COVID-19 también está afectando de manera muy importante a las mujeres, por su sobrecarga en las tareas de cuidado y al encontrarse sobrerrepresentadas en las actividades económicas en las que las medidas de aislamiento preventivo han tenido más impacto. Los pueblos indígenas y afrodescendientes también se verán más afectados por la crisis, dado que enfrentan peores condiciones socioeconómicas y tienen un acceso más limitado a la oferta pública que el resto de la población, a lo que se suman procesos históricos de discriminación (CEPAL/OPS, 2020). 
- La enfermedad por coronavirus (COVID-19) llegó oficialmente a la región el 26 de febrero, fecha en que se anunció el primer caso en el Brasil, y desde entonces los países de América Latina y el Caribe se fueron sumando gradualmente a las medidas que se estaban tomando en otras partes del mundo, como la limitación del transporte en todas las vías a lo estrictamente esencial para atender la pandemia, la suspensión de la participación presencial en centros educativos y oficinas, el confinamiento de la población en sus hogares y la promoción del distanciamiento físico (OMS, 2020b). En agosto de 2020, la mayoría de los países de la región aún mantenían medidas como el cierre de fronteras y del comercio internacional, el aislamiento obligatorio y la suspensión de múltiples sectores económicos (OCDE, 2020), lo cual ha prolongado las consecuencias negativas para la seguridad y la estabilidad del ingreso familiar (CEPAL/OIT, 2020) y para la salud mental de padres, madres y cuidadores (Anderson y otros, 2020).

\section{A. Los efectos socioeconómicos de la crisis exacerban los riesgos}

- Ante la llegada de la pandemia y sus posteriores implicaciones, América Latina y el Caribe no contaba con la suficiente preparación para un reto que tan solo se veía venir a finales del primer trimestre del año, a pesar de haber tenido mayor anticipación que los países del hemisferio norte. En un tiempo récord, los Gobiernos tuvieron que disponer de

Las niñas, niños y adolescentes no son la cara de esta pandemia, pero corren el riesgo de convertirse en sus principales víctimas.

UNICEF (2020b, pág.2) recursos sin precedentes y emplearlos en medidas para reforzar el sector de la salud y proveer protección social a amplios sectores de la población (CEPAL, 2020c). Para contener la difusión de los contagios y evitar la saturación de los sistemas de salud, los Gobiernos de América Latina y el Caribe debieron imponer estrictas normas de distanciamiento físico que conllevaron la disminución de la actividad económica interna. A los factores internos se sumó la disminución del comercio internacional, la caída de los precios de los bienes primarios, la interrupción de las cadenas globales de valor, una menor demanda en el sector de viajes y turismo y el empeoramiento de las condiciones financieras mundiales (CEPAL, 2020a).

- Se prevé que la crisis del COVID-19 aumentará la pobreza. Además de la inseguridad en el ingreso familiar, las restricciones a la libre movilidad, el aislamiento y el hacinamiento incrementan considerablemente el estrés y la ansiedad en padres, madres y cuidadores, así como en niñas, niños y adolescentes (OMS, 2020a). Si bien las medidas de aislamiento han sido una respuesta adecuada ante una pandemia sin precedentes, al contribuir a contener la propagación del virus en la mayoría de los países, las capacidades para recuperarse de los efectos sociales y económicos de dichas medidas son dramáticamente diferentes entre los países desarrollados y los países en desarrollo, como los de América Latina y el Caribe (Cornia, Jolly y Stewart, 2020). Esto se debe a la ausencia, en estos últimos, de sistemas de protección social que puedan garantizar el bienestar de las personas durante la suspensión de la actividad económica, lo que contrasta con el mayor desarrollo y capacidad en infraestructura, tecnología y cualificación de personal, entre otros, de los países desarrollados, que no solo les permitieron enfrentar la crisis más rápidamente con menos recursos (o mayor capacidad de inversión), sino que además facilitarán la inversión pública necesaria para intervenir en los frentes afectados por la pandemia (CEPAL, 2020b).

- La CEPAL (2020d) proyecta que en América Latina el número de personas en situación de pobreza se incrementará 45,4 millones en 2020 , con lo que el total de personas en situación de pobreza pasaría de 185,5 millones en 2019 a 230,9 millones en 2020, cifra que representa el $37,3 \%$ de la población latinoamericana. Dentro de este grupo, el número de personas en situación de pobreza extrema aumentaría 28,5 millones, pasando de 67,7 millones de personas en 2019 a 96,2 millones de personas en 2020, cifra que equivale al 15,5\% del total de la población.

- En América Latina y el Caribe, la recesión más profunda en décadas —se proyecta una contracción del PIB del 9,1\% (CEPAL, 2020d) — se manifestará en la contracción de la mayoría de los mercados, como se espera que suceda en un gran número de países emergentes (Banco Mundial, 2020). Se estima que la población infantil en situación de pobreza monetaria en América Latina podría incrementarse hasta 7,6 puntos porcentuales, de forma que se vería afectado el 51,3\% de esta población (es decir, uno de cada dos niños, niñas y adolescentes) (CEPAL/UNICEF, 2020). Esto irá de la mano con el agravamiento de la crisis de la educación; el aumento de los desafíos para la salud, el desarrollo y la supervivencia, y la exposición al riesgo de sufrir violencia y otras vulneraciones (UNICEF, 2020b). 
- Las personas que habitan en hogares con pobreza multidimensional aguda tienen privaciones severas en áreas fundamentales para la supervivencia (alimentación adecuada, acceso a servicios públicos, escolaridad o una vivienda adecuada) que afectan principalmente a niñas, niños y adolescentes (Alkire y otros, 2020; Santos, 2020). Por otra parte, y aunque en ocasiones la cohabitación entre hogares puede ser funcional o incluso favorable para el bienestar colectivo, el número excesivo de personas que ocupan el recinto de vivienda puede dar lugar a problemas de convivencia, violencia doméstica y falta de privacidad (ONU-Hábitat, 2015), que constituyen riesgos de violencia sexual.

- A la precariedad de las condiciones socioeconómicas que afrontan las familias, se añade el hecho de que las instituciones educativas de América Latina y el Caribe, al igual que las de más de 190 países de todo el mundo, cancelaron las clases presenciales y dejaron fuera de la escuela a estudiantes de todos los niveles de enseñanza (UNESCO, 2020; CEPAL/UNESCO, 2020). Si bien esta medida ha permitido el distanciamiento físico necesario para contener el contagio (OMS, 2020b), confinó a buena parte de las niñas, niños y adolescentes escolarizados en sus hogares, con lo que desprotegió a los millones de ellos que dependían de la escuela y los programas sociales asociados para cubrir otras necesidades, como la alimentación o la atención a la primera infancia (UNICEF, 2020b). Esta medida limitó drásticamente las posibilidades del personal docente de detectar y denunciar casos de violencia, de brindar acompañamiento y asesoría y de activar rutas de respuesta (Peterman, O'Donnell, Palermo, 2020). Esto se sumó a la medida tomada en la mayoría de los países (OCDE, 2020) de priorizar el uso presencial de los servicios de salud para casos relacionados con el COVID-19, que limitó a su vez la posibilidad de detección de posibles casos de violencia por parte del personal de la salud.

\section{B. Más tiempo en Internet sin el adecuado acompañamiento y supervisión: más riesgo}

- El entorno digital ha permitido, para quienes tienen acceso a él, mantener activos, entre otros, los sectores educativo y laboral, interactuar con la burocracia del Estado y relacionarse con familiares y amigos. Buena parte de la vida comunitaria, el acceso a servicios y trámites públicos, la interacción social, el trabajo y el estudio remotos y el entretenimiento se trasladaron a los entornos digitales (UNICEF, 2020b). Sin embargo, se estima que solo cuatro de cada diez hogares en América Latina y el Caribe tienen acceso a Internet de banda ancha y que el acceso a Internet móvil no supera el 50\% (CAF, 2020). Este acceso limitado tiene repercusiones negativas para las intervenciones que, antes de la pandemia, se realizaban con visitas domiciliarias y desde los centros de desarrollo infantil y que ahora han sido reemplazadas por un acompañamiento a distancia. Adicionalmente, la falta de alfabetización digital y la brecha de conocimiento entre personas adultas y niñas, niños y adolescentes (CAF, 2020) puede limitar la labor de acompañamiento de padres, madres y cuidadores en las tareas relacionadas con la educación a distancia y, en el caso de las niñas y niños menores de cinco años, en las labores de cuidado cariñoso y sensible realizadas en guarderías o centros de cuidado infantil (OMS, 2018).

- Los niños, niñas y adolescentes de familias que se encuentran en cuarentena pasan una cantidad de tiempo considerable en los entornos digitales para estudiar, socializar y jugar (End Violence Against Children, 2020a). Este tiempo en línea aumenta su exposición a personas que puedan abusar de ellos por Internet (End Violence Against Children, 2020b). Esto se debe a la gran cantidad de espacios digitales en que transcurre el tiempo de ocio durante el aislamiento, que incluyen juegos y comunidades virtuales donde es posible interactuar abiertamente con jugadores desconocidos (Centre for Sport and Human Rights, 2020), entre los que se encuentran potenciales agresores que usan esta herramienta para captar a sus víctimas de manera subrepticia (INTERPOL, 2020; EUROPOL, 2020). 


\section{C ¿Por qué aún no se conoce con precisión la magnitud del problema?}

- Al cierre de agosto de 2020, es aún muy pronto para saber en detalle en qué medida y de qué forma han cambiado la violencia contra las mujeres y la violencia contra la niñez y adolescencia en el hogar y otros entornos durante la crisis del COVID-19. Antes del inicio de la pandemia ya existían desafíos para dimensionar las diferentes formas de violencia, no solo a causa de la dificultad de detección de los casos (en parte por la naturalización de la violencia, que hace que víctimas, agresores y testigos no la vean como un problema), sino también porque en muchos países aún deben crearse o mejorarse los sistemas de información (OMS, 2020a). La mayor parte de la información con que se cuenta corresponde a registros administrativos en cuyos datos no es posible medir prevalencias, aún menos al tratarse de datos de fuentes limitadas y de solo unos meses (Peterman, O'Donnell y Palermo, 2020). Se espera que la verdadera información sobre la violencia que se está produciendo durante el aislamiento en la región se conozca varios meses después de que se hayan retirado las medidas de restricción de la movilidad, pero el pronóstico unificado de diversas voces y sectores es que su magnitud y severidad crecerán con respecto al período anterior a la pandemia (OMS, 2020a).

- El aumento de los casos de violencia se refleja principalmente en el incremento de los registros administrativos de casos denunciados en plataformas de reporte y atención, en su mayoría de violencia en la pareja, sobre todo contra mujeres (Peterman y otros, 2020b). En la práctica, este incremento de los registros puede explicarse por razones diferentes al aumento real de casos, tales como la eficacia de las estrategias de comunicación durante la crisis para promover el uso de mecanismos específicos de reporte. Por esta razón, las fuentes como los registros administrativos son imprecisas a la hora de determinar la magnitud del problema; se limitan al conteo mensual de los casos que se conocen $-\mathrm{y}$ no necesariamente de la respuesta que obtuvieron- y no tienen en cuenta factores como la diferencia entre las cifras máximas de distintas temporadas o el subregistro por los casos que se dejaron de detectar en las escuelas y centros de salud (Baron, Goldstein y Wallace, 2020). Ahora bien, se ha comprobado que la detección puede disminuir en comparación con los meses previos a una crisis como la del COVID-19, como ha sucedido en otras emergencias en el mundo (Seddighi y otros, 2019). En países como los Estados Unidos, se estima que las denuncias de violencia contra la niñez disminuyen más del $60 \%$ cuando no asisten a la escuela (Fitzpatrick, Benson y Bondurant, 2020) y se observa una tendencia similar en la violencia contra las mujeres cuando no es posible detectar los casos en los lugares de trabajo o en la interacción con la familia extensa o personas de la comunidad (Peterman, O'Donnell y Palermo, 2020a). En otras palabras, las cifras no hablan de una disminución o un aumento de casos, sino de una disminución de la capacidad de detección.

\section{Los riesgos exacerbados durante la crisis del COVID-19}

- Es importante resaltar que ni el COVID-19 ni las medidas para su contención generan la violencia que se perpetra contra niñas, niños y adolescentes. La actual crisis ha exacerbado los factores de riesgo y deteriorado los factores de protección de manera que, en las condiciones actuales, las niñas, niños y adolescentes de la región están más expuestos que nunca a ser víctimas de violencia física o psicológica grave (como, por ejemplo, el uso del castigo físico y humillante como práctica de crianza), negligencia, violencia sexual y violencia en línea.

- La convergencia de estos factores genera a su vez condiciones para que los casos de violencia física, sexual y psicológica aumenten aún más (Cornia, Jolly y Stewart, 2020). El aumento de los factores de estrés, como los socioeconómicos y los relacionados con la vida familiar en aislamiento, tienen consecuencias psicológicas que pueden sobrecargar a las personas adultas, afectar su habilidad para responder a las necesidades de niñas, niños y adolescentes, y llevarlos a responder de forma agresiva (Center for the Developing Child, Harvard University, 2016 citado en Cuartas, 2020). A su vez, los datos empíricos sobre anteriores pandemias y otras emergencias permiten inferir que las mujeres y niñas están particularmente expuestas al riesgo de sufrir agresiones sexuales de forma sistemática durante su aislamiento (Peterman y otros, 2020b; Seddighi y otros, 2019; Bandiera y otros, 2018; Anderberg y otros, 2016). 
- En el marco de esta crisis, existen dos grandes amplificadores de riesgo de la violencia sexual: el ya mencionado cierre o reducción de la atención presencial en los entornos habituales de detección de casos, como las escuelas y los centros de salud, y el incremento de las horas que niñas, niños y adolescentes y los agresores pasan conectados en línea (INTERPOL, 2020; EUROPOL, 2020). En muchos casos, los niños, niñas y adolescentes incluso conviven con potenciales agresores en el hogar y el confinamiento hace que pasen más tiempo juntos. En otras palabras, hay una ventana más amplia de exposición, más riesgos y menos capacidad para detectar los casos y brindar apoyo a las víctimas (UIT, 2020). Además, aprovechando el aumento de los factores de vulnerabilidad y del número de niñas, niños y adolescentes expuestos al riesgo, los agresores se están adaptando para captar víctimas en línea y en entornos diferentes al digital (UNODC, 2020). Cuerpos de policía criminal y organismos especializados de todo el mundo coinciden en que se ha detectado un incremento de los delitos sexuales contra la niñez y adolescencia durante la pandemia de COVID-19; al dificultarse la forma habitual de acceder a sus víctimas, la mayoría de los agresores recurren a las facilidades que ofrecen las herramientas digitales (ECPAT International, 2020; INTERPOL, 2020).

- En tiempos de COVID-19, a los agresores sexuales les resulta más fácil que nunca obtener, descargar, producir y compartir material de abusos sexuales contra niñas, niños y adolescentes (ECPAT International, 2020). Para ello se valen de redes abiertas, la web profunda (deep web), la Internet oscura (dark net) y redes entre pares (peer to peer) (EUROPOL, 2020). Así, las autoridades de policía criminal

“Tenemos información de páginas web en las que los agresores discuten cómo sacar provecho de la situación que estamos viviendo".

Anna Karin Hildingson Boqvist (ECPAT, 2020) han detectado un aumento de la actividad relacionada con la violencia sexual hacia niñas, niños y adolescentes. Esto ha quedado patente en el mayor número de intentos de acceder a sitios web bloqueados por presentar contenidos de este tipo y en el incremento del intercambio de material audiovisual en comunidades virtuales de agresores y de las conversaciones en foros sobre las oportunidades de explotación y abuso sexual en las circunstancias actuales (EUROPOL, 2020).

\section{La protección de la niñez y adolescencia como prioridad} de la nueva agenda

- El retorno gradual a la actividad ha centrado la atención de las personas en el futuro inmediato. En la elaboración de este documento ha resultado evidente la falta de información sobre lo que va a suceder en la región y en el mundo a corto y largo plazo, y todavía faltan demasiados elementos de la ecuación para poder hacer pronósticos acertados. Esto alimenta la desesperanza, el estrés y la ansiedad generalizada entre las personas adultas, pero también entre niñas, niños y adolescentes. Sus necesidades deben ser aún más prioritarias, y sin embargo en muchas ocasiones han sido a quienes menos se ha tenido en cuenta. Sin duda, los países deben emprender un diálogo sobre el mundo pospandemia; la actual coyuntura es una ocasión ideal para que se dialogue con niñas, niños y adolescentes sobre qué quieren y qué no en el mundo que les quedará. Esto no solo permitirá conocer sus impresiones y aplicarlas en la reconstrucción, sino que aportará a la creación de objetivos comunes de sociedad y a disminuir la ansiedad causada por la incertidumbre sobre el futuro.

- Algunos Gobiernos han llevado a cabo acciones específicas para enfrentar la violencia contra niñas, niños y adolescentes, como la expansión de las líneas telefónicas de ayuda, las campañas de difusión de información, el financiamiento a albergues para sobrevivientes y la mejora de las condiciones de acceso a los servicios por parte de estos, así como modificaciones a los marcos normativos en materia de familia y justicia (Guedes, Peterman y Deligiorgis, 2020). Estas medidas deben aplicarse de forma sistemática y reforzarse si han de reducir la violencia contra la niñez durante y después de la crisis del COVID-19.

- En ese sentido, y en seguimiento al Ilamado del Secretario General de las Naciones Unidas a redoblar los esfuerzos para proteger a la niñez durante la pandemia, las principales organismos del sistema de las Naciones Unidas con competencia en la materia han diseñado una Agenda para la Acción contra la violencia hacia la niñez y la adolescencia en la que se trazan tres grandes 
líneas de trabajo en la contención de la violencia durante y después de la crisis: más información (la producción de datos confiables y su diseminación adecuada); mayor solidaridad para la protección de los más vulnerables, y más medidas multisectoriales en favor de los derechos de la infancia (Grupo de Trabajo Interinstitucional sobre la Violencia contra los Niños, 2020).

- Esta Agenda para la Acción debe traducirse cuanto antes en acciones concretas en los países de América Latina y el Caribe. Es evidente que la atención a la pandemia ha requerido un esfuerzo fiscal sin precedentes en los países de la región y del mundo, por lo que las acciones para enfrentar la violencia en el marco de la crisis deberán enmarcarse en inversiones públicas que ya estén en ejecución o en intervenciones que requieran un mínimo esfuerzo fiscal.

- A continuación se plantean recomendaciones de acciones para abordar la violencia contra niñas, niños y adolescentes en el marco de la respuesta a la crisis del COVID-19 que se adaptan a las posibilidades fiscales de los países de la región. El alcance de estas recomendaciones no solo se extiende al período de aislamiento, sino también al retorno gradual a la actividad. La mayoría de ellas permiten ajustar las acciones a la oferta existente o atacar varios frentes al mismo tiempo:

\section{A. La atención a la violencia contra niñas, niños y adolescentes como prioridad en la agenda de crisis de los Estados y su inclusión en las instancias intersectoriales de alto nivel creadas para responder a la crisis del COVID-19}

- En los distintos países se han constituido altas instancias de coordinación interinstitucional presididas por Jefes de Estado y sus gabinetes que se han mostrado eficaces a la hora de poner todos los recursos de los Gobiernos y sus aliados a disposición de resolver la crisis del COVID-19. Esto ha permitido asignar recursos a asuntos que van desde el mejoramiento contrarreloj de la infraestructura de salud hasta ayudas financieras a personas y sectores afectados por las consecuencias socioeconómicas de la crisis.

- La Agenda 2030 para el Desarrollo Sostenible insta a los Estados a prevenir y eliminar la violencia contra niñas, niños y adolescentes (meta 16.2 de los Objetivos de Desarrollo Sostenible), una cuestión imperativa para la sostenibilidad de los países. Este compromiso cobra mayor fuerza en el contexto de la crisis del COVID-19, independientemente de que existiera o no en los países un compromiso público o un plan específico para abordar dicha violencia ya antes de la pandemia. Dada la gravedad del problema y sus implicaciones, debe plantearse — desde el liderazgo de los Jefes de Estado y sus gabinetes- en las altas instancias de coordinación interinstitucional mencionadas, donde habrían de coordinarse planes que se alimenten de recursos técnicos, financieros y de talento humano procedentes de todos los actores, y no solo de las entidades encargadas de la protección de niñas, niños y adolescentes.

- De igual manera, es importante garantizar que los servicios disponibles para sobrevivientes de la violencia se mantengan abiertos, y que se considere a las personas que trabajan en ellos personal esencial, que tengan acceso a los equipamientos necesarios de bioseguridad y que estén capacitadas para atender casos según el género y la edad.

\section{B. Optimización de los recursos mediante la convergencia de la oferta de servicios}

- Uno de los factores de éxito en la respuesta de los países de la región a la crisis ha sido la convergencia de la oferta. El marco estratégico INSPIRE ${ }^{5}$ para la eliminación de la violencia contra la niñez y adolescencia se diseñó sobre la base de experiencias exitosas, y demuestra que la eliminación de la violencia es posible desde un abordaje intersectorial coordinado. Sin embargo, para responder a la crisis del COVID-19 los países se han visto obligados a tomar medidas inmediatas, que no siempre estaban enmarcadas en políticas y estrategias existentes.

- A través de las instancias de coordinación mencionadas se ha logrado que diversas carteras pongan a disposición presupuestos y programas para dar una respuesta conjunta. Esto permite aunar la experticia y experiencia propia de cada institución pública y privada en iniciativas conjuntas, optimizar recursos en tiempos de austeridad fiscal y abordar varias áreas con acciones integrales. Estas acciones pueden incluir:

\footnotetext{
5 Véase más información en [en línea] https://www.end-violence.org/inspire.
} 
1. Convergencia de la respuesta ante la violencia contra las mujeres y contra niñas, niños y adolescentes:

- La respuesta a la crisis ha incluido la creación o el fortalecimiento de los servicios de atención a los casos de violencia contra las mujeres en el hogar, como las líneas telefónicas de apoyo y denuncia, aunque estos servicios aún deben fortalecerse más. Como se expuso en apartados anteriores, ha quedado demostrada la conveniencia de intervenir con acciones complementarias en la prevención de la violencia contra las mujeres y la violencia contra niñas, niños y adolescentes; comparten factores de riesgo y las medidas para contrarrestar una impactarían directamente sobre la otra. Para ello es importante generar espacios de coordinación que permitan usar recursos técnicos, financieros y de infraestructura comunes, y a la vez aprovechar la experticia del talento humano que trabaja en cada uno de los temas. Algunas medidas podrían ser facilitar el acceso de sobrevivientes a puntos de reporte especiales, como se ha hecho en el caso de las mujeres sobrevivientes de violencia (con la instalación de puntos de apoyo en los mercados - la capacitación a farmacéuticos), establecer aplicaciones y líneas de ayuda específicas para niñas, niños y adolescentes, o habilitar albergues de emergencia para mujeres sobrevivientes de violencia en el hogar en los que se pueda dar alojamiento también a sus hijos e hijas.

Alojamiento seguro en hoteles para mujeres, niñas y niños víctimas de violencia en México

En respuesta al aumento de la violencia intrafamiliar generado en el contexto del COVID-19, el UNICEF, el Fondo de Población de las Naciones Unidas (UNFPA), el Gobierno de México y el sector hotelero han iniciado la primera fase de una estrategia de alojamiento seguro y gratuito en hoteles para mujeres y sus hijas e hijos víctimas de violencia mientras se estudian sus casos y se localizan diferentes redes de apoyo o refugios.

Antes del distanciamiento físico impuesto por la pandemia, el hogar ya era un lugar peligroso para las mujeres y sus hijas e hijos. Por ejemplo, según las encuestas, el $44 \%$ de las mujeres había experimentado algún tipo de violencia por parte de su actual o última pareja. Asimismo, 6 de cada 10 niñas o niños de entre 1 y 14 años habían experimentado alguna medida disciplinaria violenta en sus hogares, según datos de la Encuesta Nacional de Niños, Niñas y Mujeres (ENIM) de 2015.

Adicionalmente, entre enero y junio de 2020 las llamadas de emergencia por incidentes relacionados con violencia contra la mujer aumentaron un $45,8 \%$ en comparación con el mismo semestre de 2019. Estudios previos muestran que la violencia contra las mujeres en el hogar afecta igualmente a niñas, niños y adolescentes, por el hecho de ser testigos de esta y porque, en la mayoría de las ocasiones, la persona agresora también ejerce violencia en su contra.estamos viviendo.

2. Ampliación del número de receptores de servicios públicos de ayuda durante la pandemia de COVID-19

- Aunque en un principio haya podido suponer una inversión pública adicional, la mayoría de los países de la región han puesto en marcha programas como el refuerzo de las transferencias monetarias y la entrega de canastas alimentarias a amplios sectores de la población. El retorno de esta inversión y de las intervenciones complementarias se verá en la prevención a mediano plazo de situaciones de alto costo social cuya resolución requeriría una inversión mayor. En la misma línea, también es importante reducir las barreras de acceso a la oferta de servicios y eliminar las condiciones exigidas para que las familias, y específicamente las mujeres, puedan acceder a las ayudas.

3. Intervenciones específicas y diferenciales en el caso de niñas, niños y adolescentes que ya estaban en situación de vulnerabilidad

- Desde antes de la crisis del COVID-19, los niños, niñas y adolescentes en situación de vulnerabilidad ya eran una población sobre la que no siempre se contaba con suficiente información para incluirla en la oferta estándar del Estado. Si bien es importante generar estrategias diferenciales que aborden sus necesidades particulares, el hecho de incluirlos también en la oferta regular ayudará a canalizar otras ayudas que mitiguen los riesgos inherentes a su condición de vulnerabilidad. Las intervenciones específicas y diferenciales reducirán la vulnerabilidad adicional que enfrentan grupos poblacionales que en ocasiones se encuentran fuera del radar de la oferta social de los Estados: niñas, niños y adolescentes refugiados, apátridas, migrantes, desplazados, en situación de calle, privados de libertad, institucionalizados, indígenas, afrodescendientes o que ya están siendo víctimas de vulneraciones como trabajo infantil o que se encuentran en situaciones de conflicto y fragilidad (UNICEF, 2020e). 


\section{Promoción de la economía del cuidado y del equilibrio en la distribución de roles y tareas}

- El paso de las clases presenciales al ámbito virtual y el nuevo formato remoto de los programas sociales han llevado a niñas, niños y adolescentes a depender más que nunca de sus padres, madres o cuidadores para recibir las atenciones necesarias para su desarrollo y para cumplir con sus obligaciones escolares, así como para participar en actividades de ocio y otros programas públicos de los que ya eran beneficiarios. Esto ha generado un efecto positivo inesperado: en muchos casos se ha incrementado

La pandemia del COVID-19 profundiza la crisis de los cuidados en América Latina y el Caribe (CEPAL, (2020e). Véase más información sobre cómo las desigualdades de género se han acrecentado en los hogares durante la pandemia: [en línea] https://repositorio. cepal.org/bitstream/handle/11362/ 45335/5/S2000261_es.pdf la participación de todos los miembros del hogar en las actividades de niñas, niños y adolescentes, pero también supone una carga adicional en tiempo y dedicación. En ese sentido, en el caso de las familias en situación de vulnerabilidad, podrían ofrecerse estímulos monetarios o en especie (como subsidios alimentarios complementarios o exenciones en el pago de servicios) que reconozcan el tiempo dedicado y potencien el rol activo de los familiares. Sin embargo, teniendo en cuenta que aún es necesario avanzar en la promoción de la distribución equitativa de roles y tareas, estas acciones deben, además, propiciar la participación activa del padre y de los demás hombres del núcleo familiar.

\section{Transformación de prácticas}

- Los esfuerzos sin precedentes para hacer frente a la pandemia de COVID-19 han incluido estrategias de comunicación eficaces para transformar rápidamente los hábitos de las personas y, en solo unos meses, se han logrado transformar los hábitos de la sociedad. Gracias a campañas y estrategias reforzadas en todos los medios, se han adquirido hábitos de autocuidado que hace meses no formaban parte de la cotidianidad, como el lavado más frecuente de manos, el uso de mascarillas y tapabocas, y hasta la forma en que las personas se saludan y manifiestan afecto fuera de sus núcleos familiares cercanos. Estas estrategias se mantendrán y reforzarán con el tiempo, más aún a medida que los países regresen gradualmente a la actividad.

- Para reducir la violencia contra niñas, niños y adolescentes en el hogar, es importante incorporar conocimientos, actitudes y prácticas a los que muchas familias son ajenas. Las iniciativas mencionadas pueden servir de base, por ejemplo, para proponer escenarios de comunicación en la familia, prácticas no violentas de crianza y crianza positiva, o para sugerir actividades en los hogares en las que se incorpore la igualdad de género en la crianza.

\section{Fomento del rol activo del profesorado y del personal de los centros}

\section{de desarrollo de la primera infancia}

- La cancelación de la asistencia presencial en las instituciones educativas y centros de desarrollo de la primera infancia supuso también la supresión de una de las principales fuentes de detección de casos de violencia contra niñas, niños y adolescentes. Al mismo tiempo, los educadores debieron adaptar su interacción con al alumnado a dinámicas no presenciales. En esa medida, la reinvención de los entornos educativos y de los métodos de enseñanza y aprendizaje debe incluir el refuerzo de las habilidades socioemocionales, así como contenidos específicos en las clases sobre la violencia contra niñas, niños y adolescentes y sus causas estructurales (como las normas sociales que sustentan la violencia). Deben fortalecerse los canales directos de comunicación entre docentes, familias y estudiantes, de forma que la relación de confianza permita que niñas, niños y adolescentes informen sobre situaciones de riesgo o violencia directa o indirecta a la que se han visto expuestos. Por su parte, el apoyo a la labor docente debe incluir formación orientada al diálogo con estudiantes sobre violencia, así como el rediseño de las rutas de detección y de la respuesta ante casos detectados. En el caso de las instituciones que cuenten con personal psicopedagógico, resultará clave su participación activa tanto en la construcción de rutas y protocolos como en la atención y derivación de los posibles casos identificados. 


\section{Oferta psicosocial desde la prevención}

- Se ha considerado la atención de la salud mental como una de las acciones necesarias para dar respuesta a los casos de violencia detectados. Sin embargo, es importante que la oferta de atención psicosocial sea abierta y que no se condicione a haber sido víctima de violencia, tanto en el caso de niñas, niños y adolescentes como en el de las mujeres. Esto significa habilitar servicios de atención psicosocial para la población en general, que permitan prevenir y atender trastornos de salud mental como la depresión, la ansiedad, la ideación suicida y el estrés en padres, madres y cuidadores yen los propios niños, niñas y adolescentes. Estas intervenciones deben, además, promover prácticas asertivas de crianza y de comunicación en el hogar. Aunque varios países han dispuesto portales y otros mecanismos de información sobre estas, es importante reconocer la importancia de la intervención en la salud mental caso por caso, además de las actividades e información complementaria de acceso general. A la hora de ofrecer estos servicios, es importante poner en marcha medios alternativos, tales como la atención vía telefónica o a través de medios digitales, que salvaguarden la privacidad y la confidencialidad.

En la República Dominicana, el Fondo de las Naciones Unidas para la Infancia (UNICEF), el Colegio Dominicano de Psicólogos y el Instituto de Salud Mental y Telepsicología crearon la Línea de Apoyo Emocional a Familias "Contigo".

Esta línea de ayuda está disponible las 24 horas del día, los siete días de la semana y ofrece, de manera gratuita y remota, primeros auxilios psicológicos, apoyo emocional, psicoeducación y derivación a servicios especializados (violencia contra niñez y adolescencia, violencia intrafamiliar, violencia de género, riesgo suicida y riesgo psiquiátrico).

Estos servicios están disponibles en español, creole haitiano y lengua de señas, a través de llamadas telefónicas; llamadas y chat por WhatsApp, y chat a través de su página web [en línea] www.lineafamiliar.do.

\section{Incremento del acceso real de la población a las tecnologías de la información y las telecomunicaciones}

- Las TIC, en especial los entornos digitales, han sido importantes tanto para la Administración como para las relaciones sociales en la crisis del COVID-19. Si bien las TIC han permitido que los Gobiernos puedan llegar a sus beneficiarios, las circunstancias también han exacerbado los efectos de la brecha digital. Por esta razón, es importante:

- Emplear también medios tradicionales, como la radio abierta y las emisoras comunitarias, para llegar a la población en lugares con conectividad limitada. Esto implica crear estrategias diferenciales diseñadas para estos medios que no se limiten a campañas de información con mensajes estándar.

- Establecer alianzas con el sector privado de la tecnología y las telecomunicaciones para aumentar la penetración de Internet en las áreas no cubiertas, así como la distribución de dispositivos electrónicos a las familias.

- Poner en marcha programas de formación en habilidades digitales para padres, madres y cuidadores que les permitan adquirir conocimientos y habilidades para conocer uno de los entornos en que más horas pasan niñas, niños y adolescentes en muchos hogares.

- Si bien las estrategias de promoción del autocuidado por parte de niñas, niños y adolescentes son importantes y deben mantenerse y reforzarse, las estrategias de prevención de la violencia en el entorno digital deben incluir de forma más activa a los padres, madres y cuidadores. De esta manera, la propia familia puede informar a los niños, niñas y adolescentes sobre los riesgos a los que se exponen con un uso inadecuado de Internet. Este punto depende de una adecuada y continua formación sobre el ámbito digital.

- Fomentar la creación de herramientas (aplicaciones u otras) que faciliten el control parental en los dispositivos que usan niñas, niños y adolescentes para relacionarse en el entorno digital. De esta manera se puede reducir la posibilidad de contacto con potenciales agresores y el acceso a contenidos no adecuados para su edad.

- Promover estrategias de regulación de la oferta de contenidos y plataformas, de cara a proteger los derechos de niñas, niños y adolescentes, con la participación activa del sector privado. 


\section{E. Acciones de alto impacto en el entorno laboral sin costos mayores para los empleadores}

- Los riesgos relacionados con la pandemia de COVID-19 han obligado a replantear las dinámicas en los entornos laborales, lo que, por otro lado, supone una oportunidad para llevar a cabo ajustes que permitan erradicar algunas de las causas estructurales de la violencia contra niñas, niños y adolescentes y contra las mujeres. Al respecto, la CEPAL ha destacado la forma en que las políticas con enfoque de género pueden reducir las desigualdades de género en los hogares y en los entornos laborales, así como nivelar la distribución de los roles de cuidado en

El sector privado puede aportar de manera significativa a la prevención de la violencia en el hogar y otros entornos. Véase más información sobre cómo las empresas pueden participar activamente: [en línea] https://violenceagainstchildren. un.org/sites/violenceagainstchildren. un.org/files/el_sector_privado_y_la_ prevencion_de_la_violencia_contra_ los_ninos_y_ninas_en_lac_-_esp.pdf el hogar (CEPAL, 2020e). Para ello, se requiere avanzar en la autonomía de las mujeres en el entorno de trabajo, lo cual contribuirá a reducir efectos como el desequilibrio en las relaciones de poder, que pueden propiciar la violencia en el hogar (Vaca Trigo, 2019). En el marco de lo que se han denominado políticas favorables a la familia, el UNICEF ha propuesto una serie de medidas para diseñar lugares de trabajo que incrementen la eficiencia de sus colaboradores y equilibren adecuadamente el bienestar de sus familias (UNICEF, 2019). Estas medidas están orientadas a favorecer la participación de los hombres en la crianza de sus hijas e hijos, la adecuación de espacios para madres gestantes y lactantes y la reducción del estrés en madres y padres. Las políticas favorables a la familia pueden asegurar un equilibrio adecuado entre la vida laboral y familiar de las personas adultas. Entre estas políticas se incluyen la licencia parental remunerada, la licencia médica para el cuidado de hijas e hijos convalecientes, la adecuación de guarderías en los lugares de trabajo, horarios laborales flexibles y prestaciones por cada hijo.

\section{F. Creación de soluciones y oportunidades a nivel local y comunitario con la participación real de niñas, niños y adolescentes}

- A pesar de que las condiciones del aislamiento y del retorno gradual a la actividad limitan la interacción real de los niños, niñas y adolescentes con los miembros de la comunidad y con familiares con los que no conviven, estas condiciones, tal y como se ha expuesto, generan un riesgo adicional frente a la violencia. Desde las administraciones locales y comunitarias es posible poner en marcha, con los medios disponibles, mecanismos de interacción virtuales o que respeten el distanciamiento físico mínimo y que permitan que la cotidianidad de las personas transcurra más allá de la intimidad de los hogares. Para esto pueden promoverse entornos de videoconferencia gratuitos en las comunidades, así como servicios de telecomunicaciones (como llamadas por teléfono celular) gratis entre los miembros de la familia. De igual manera, es importante destacar la función y las posibilidades de llegar de una forma más efectiva a los beneficiarios de los programas a través de las autoridades locales, de las autoridades tradicionales de los pueblos originarios y de los líderes de las comunidades espirituales. 


\section{Bibliografía}

Alianza para la Protección de la Infancia en la Acción Humanitaria (2020a), Technical Note: Protection of Children during the Coronavirus Pandemic (v. 1), mayo.

(2020b), COVID-19 and its Implications for Protecting Children Online, Ginebra, abril.

Alkire, S. y otros (2020), "Multidimensional poverty and COVID-19 risk factors: a rapid overview of interlinked deprivations across 5.7 Billion People", OPHI Briefing, vol. 53, Oxford Poverty and Human Development Initiative, Universidad de Oxford.

Anderberg, D. y otros (2016), "Unemployment and domestic violence: theory and evidence", The Economic Journal, 1947-1979.

Anderson, R. M. y otros (2020), "How will country-based mitigation measures influence the course of the COVID-19 epidemic?", The Lancet, vol. 395.

Banco Mundial (2020), "Global economic prospects, June 2020", A World Bank Group Flagship Report, Washington, D.C.

Bandiera, O. y otros (2018), "The economic lives of young women in the time of Ebola: lessons from an Empowerment Program", World Bank Policy Research Working Paper, № 8760.

Baron, E., E. Goldstein y C. Wallace (2020), Suffering in Silence: How COVID-19 School Closures Inhibit the Reporting of Child Maltreatment, 14 de mayo [en línea] https://ssrn.com/abstract=3601399 o http:// dx.doi.org/10.2139/ssrn.3601399.

Bhatia, A. y otros (2020), Comment: Beyond children as "invisible carriers":The implications of COVID-19response measures on violence against children [en línea] https://www.who.int/bulletin/volumes/98/9/20-263467/en/.

Blackman, A. y otros (2020), La política pública frente al COVID-19: recomendaciones para América Latina y el Caribe, Washington, D.C., Banco Interamericano de Desarrollo (BID).

Bott, S. y A. Ruiz-Celis (2019), "Intersecciones entre violencia contra las niñas y niños y violencia contra las mujeres en América Latina y el Caribe", inédito.

CAF (Banco de Desarrollo para América Latina) (2020), El estado de la digitalización de América Latina frente a la pandemia del COVID-19. Observatorio CAF del Ecosistema Digital, abril [en línea] https:/l scioteca.caf.com/bitstream/handle/123456789/1540/EI_estado_de_la_digitalizacion_de_America_ Latina_frente_a_la_pandemia_del_COVID-19.pdf?sequence=1 \&isAllowed=y.

Centre for Sport and Human Rights (2020), An Overview of the Sport-Related Impacts of the COVID-19 Pandemic on Children, Ginebra.

CEPAL (Comisión Económica para América Latina y el Caribe) (2020a), "América Latina y el Caribe ante la pandemia del COVID-19: efectos económicos y sociales", Informe Especial COVID-19, № 1, Santiago, 3 de abril.

(2020b), "El desafío social en tiempos del COVID-19", Informe Especial COVID-19, №3, Santiago, 12 de mayo. (2020c), "Dimensionar los efectos del COVID-19 para pensar en la reactivación", Informe Especial COVID-19, № 2, Santiago, 21 de abril.

_ (2020d), "Enfrentar los efectos cada vez mayores del COVID-19 para una reactivación con igualdad: nuevas proyecciones", Informe especial COVID-19, N 5, Santiago, 15 de julio.

(2020e), La pandemia del COVID-19 profundiza la crisis de los cuidados en América Latina y el Caribe, Santiago, abril de 2020.

CEPAL/FAO (Comisión Económica para América Latina y el Caribe/Organización de las Naciones Unidas para la Alimentación y la Agricultura) (2020), "Cómo evitar que la crisis del COVID-19 se transforme en una crisis alimentaria: acciones urgentes contra el hambre en América Latina y el Caribe", Informe COVID-19. CEPAL-FAO, Santiago.

CEPAL/OIT (Comisión Económica para América Latina y el Caribe/Organización Internacional del Trabajo) (2020), "El trabajo en tiempos de pandemia: desafíos frente a la enfermedad por coronavirus (COVID-19)", Coyuntura Laboral en América Latina y el Caribe, № 22 (LC/TS.2020/46), Santiago.

CEPAL/OPS (Comisión Económica para América Latina y el Caribe/Organización Panamericana de la Salud) (2020), "Salud y economía: una convergencia necesaria para enfrentar el COVID-19 y retomar la senda hacia el desarrollo sostenible en América Latina y el Caribe", Informe Especial COVID-19 CEPAL-OPS, Santiago, 30 de julio.

CEPAL/UNESCO (Comisión Económica para América Latina y el Caribe/Organización de las Naciones Unidas para la Educación, la Ciencia y la Cultura) (2020), "La educación en tiempos de la pandemia de COVID 19", Informe COVID-19 CEPAL-UNESCO, Santiago, agosto. 
CEPAL/UNICEF (Comisión Económica para América Latina y el Caribe/Fondo de las Naciones Unidas para la Infancia) (2020), "Protección social para familias con niños, niñas y adolescentes en América Latina y el Caribe: un imperativo frente a los impactos del COVID-19. Informes COVID-19", inédito.

(2018), América Latina y el Caribe a 30 años de la aprobación de la Convención sobre los Derechos del Niño (LC/PUB.2018/21), Santiago.

Cornia, G. A., R. Jolly y F. Stewart (2020), "COVID-19 and children, in the North and in the South", Innocenti Discussion Papers, April, 1-10 [en línea] https://www.unicef-irc.org/publications/1087-covid-19-andchildren-in-the-north-and-the-south.html.

Cuartas, J. (2020), "Heightened risk of child maltreatment amid the COVID-19 pandemic can exacerbate mental health problems for the next generation," Psychological Trauma: Theory, Research, Practice, and Policy [en línea] https://doi.org/10.1037/tra0000597.

_(2019), “Violencia contra niñas, niños y adolescentes: etiología, consecuencias y estrategias para su prevención", Informe para el Instituto Colombiano de Bienestar Familiar, inédito.

Cuartas, J. y otros (2019), "Early childhood exposure to non-violent discipline and physical and psychological aggression in low- and middle-income countries: national, regional, and global prevalence estimates", Child Abuse \& Neglect, vol. 92.

ECPAT International (2020), Why Children are at Risk of Sexual Abuse and Exploitation during COVID-19 [en línea] https://www.ecpat.org/news/covid-19-sexual-abuse/.

(2018), Online Child Sexual Abuse Material-The Facts [en línea] https://www.ecpat.org/news/onlinechild-sexual-abuse-material-the-facts/.

End Violence Against Children (2020a), "Stay Safe at Home. Stay Safe Online" [en línea] https://www.endviolence.org/safeonlinecovid.

_(2020b), COVID-19 and its Implications for Protecting Children Online, Ginebra, abril.

EUROPOL (2020), Exploiting isolation: Offenders and victims of online child sexual abuse during the COVID-19 pandemic, 19 de junio [en línea] https://www.europol.europa.eu/publications-documents/ exploiting-isolation-offenders-and-victims-of-online-child-sexual-abuse-during-covid-19-pandemic.

Fitzpatrick, M., C. Benson y S. Bondurant (2020), "Beyond reading, writing and arithmetic: the role of teachers and schools in reporting child maltreatment," NBER Working paper Series, № 27033.

Grupo de Trabajo Interinstitucional sobre la Violencia contra los Niños (2020), Agenda para la Acción [en línea] https://violenceagainstchildren.un.org/sites/violenceagainstchildren.un.org/files/2020/agenda_for_action lagenda_for_action_spanish.pdf.

Guedes, A., A. Peterman y D. Deligiorgis (2020), "Five Ways governments are responding to violence against women and children during COVID-19", UNICEF Connect [en línea] https://blogs.unicef.org/ evidence-for-action/five-ways-governments-are-responding-to-violence-against-women-and-childrenduring-covid-19/.

Guedes, A. y otros (2016), Bridging the gaps: a global review of intersections of violence against women and violence against children, Global Health Action [en línea] http://dx.doi.org/10.3402/gha.v9.31516.

INTERPOL (2020), "COVID-19 cyberthreats" [en línea] https://www.interpol.int/Crimes/Cybercrime/ COVID-19-cyberthreats.

Naciones Unidas (2020a), "Secretary-General's Appeal for Global Ceasefire" [en línea] https://www.un.org/ sg/en/content/sg/statement/2020-03-23/secretary-generals-appeal-for-global-ceasefire.

(2020b), Informe: El impacto del COVID-19 en América Latina y el Caribe, julio.

(2013), The Six Grave Violations against Children during Armed Conflict: The Legal Foundation, Oficina del Representante Especial del Secretario General para la Cuestión de los Niños y los Conflictos Armados (ORESG-NCA).

(2006), “Observatorio General № 8 (2006): El derecho del niño a la protección contra los castigos corporales y otras formas de castigo crueles o degradantes (artículo 19, párrafo 2 del artículo 28 y artículo 37, entre otros)" (CRC/C/GC/8), Comité de los Derechos del Niño.

Nguyen, K. y otros (2018), "Diseño del muestreo y metodología de las encuestas sobre la violencia contra niños, niñas y jóvenes" [en línea] https://www.togetherforgirls.org/wp-content/uploads/Dise\%C3\%B1odel-muestreo-y-metodolog\% C3\%ADa-de-las-Encuestas-sobre-la-Violencia-contra-Ni\% C3\%B1osNi\%C3\%B1as-y-J\%C3\%B3venes.pdf.

OCDE (Organización de Cooperación y Desarrollo Económicos) (2020), "COVID-19 in Latin America and the Caribbean: An overview of government responses to the crisis" [en línea] https://read.oecd-ilibrary.org/ view/?ref=129_129907-eae84sciov\&title=COVID-19-in-Latin-Amercia-and-the-Caribbean_An-overviewof-government-responses-to-the-crisis. 
OIT (Organización Internacional del Trabajo) (2020), Panorama Laboral 2019: América Latina y el Caribe, Lima. (1999), "Convenio sobre las Peores Formas de Trabajo Infantil, 1999 (núm. 182)", Ginebra, 17 de junio.

OIT/CEPAL (Organización Internacional del Trabajo/Comisión Económica para América Latina y el Caribe) (2020), "La pandemia por COVID-19 podría incrementar el trabajo infantil en América Latina y el Caribe", Nota Técnica, № 1, Santiago.

OMS (Organización Mundial de la Salud) (2020a), Informe sobre la situación mundial de la prevención de la violencia contra los niños de 2020, Ginebra.

_(2020b), "Coronavirus disease (COVID-19) pandemic" [en línea] https://www.who.int/emergencies/ diseases/novel-coronavirus-2019.

_. (2018), Cuidado cariñoso y sensible para el desarrollo en la primera infancia, Ginebra.

ONU-Hábitat (Programa de las Naciones Unidas para los Asentamientos Humanos) (2015), Déficit habitacional en América Latina y el Caribe: una herramienta para el diagnóstico y el desarrollo de políticas efectivas en vivienda y hábitat, Nairobi.

Peterman, A., A. Bhatia y A. Guedes (2020), Remote data collection on violence against women during COVID-19: A conversation with experts on ethics, measurement \& research priorities [en línea] https:// www.unicef-irc.org/article/1997-remote-data-collection-on-violence-against-women-during-covid-19-aconversation-with.html.

Peterman, A., M. O'Donnell yT. Palermo (2020), "COVID-19 and violence against women and children what have we learned so far?", CGD Note, Center for Global Development, junio.

Peterman, A. y otros (2020a), "Pandemics and violence against women and children", CGD Working Paper, 528, abril.

_ (2020b), "Violence unveiled: COVID-19 is shining a spotlight on domestic abuse", inédito.

Pinheiro, S. (2006), Informe mundial sobre la violencia contra los niños y niñas, Nueva York, Naciones Unidas.

Santos, M. (2020), "Pobreza multidimensional en tiempos de COVID-19" [en línea] https://www. latinamerica.undp.org/content/rblac/es/home/blog/2020/pobreza-multidimensional-en-tiempos-delcovid-19.html.

Seddighi, H. y otros (2019), "Child abuse in natural disasters and conflicts: a systematic review", Trauma, Violence \& Abuse, marzo.

Together for Girls (2020), Violence against Children Surveys [en línea] https://www.togetherforgirls.org/ violence-children-surveys/.

Tres, J. y M. Chatruc (2020), “Migrantes y COVID-19: ¿Qué están haciendo los países de América Latina con más migrantes para apoyarlos durante la pandemia?", La maleta abierta, Banco Interamericano de Desarrollo (BID) [en línea] https://blogs.iadb.org/migracion/es/migrantes-y-covid-19-que-estan-haciendolos-paises-de-america-latina-con-mas-migrantes-para-apoyarlos-durante-la-pandemia/.

Trucco, D. y A. Palma (2020), "Infancia y adolescencia en la era digital: un informe comparativo de los estudios de Kids Online del Brasil, Chile, Costa Rica y el Uruguay", Documentos de Proyectos (LC/TS.2020/18/REV.1), Santiago, Comisión Económica para América Latina y el Caribe (CEPAL).

UIT (Unión Internacional de Telecomunicaciones) (2020), Guidelines for Parents, Guardians and Educators on Child Online Protection [en línea] https://www.itu.int/dms_pub/itu-s/opb/gen/S-GEN-COP.EDUC2016-PDF-E.pdf.

UNESCO (Organización de las Naciones Unidas para la Educación, la Ciencia y la Cultura) (2020), “Education: from disruption to recovery" [en línea] https://en.unesco.org/covid19/educationresponse.

UNICEF (Fondo de las Naciones Unidas para la Infancia) (s/fa), "Ciberacoso: qué es y cómo detenerlo" [en línea] https://www.unicef.org/es/end-violence/ciberacoso-que-es-y-como-detenerlo.

_ (s/fb), "Sexual violence against children" https://www.unicef.org/protection/57929_58006.html.

_ (2020a), "Promoción del cuidado cariñoso y sensible de los niños y niñas y el autocuidado a cuidadores para prevenir la violencia en la primera infancia en tiempos de COVID-19. Marco conceptual y metodológico", por aparecer.

(2020b), Policy Brief: The Impact of COVID-19 on children, Nueva York, abril.

(2020c), A Generation to Protect: Monitoring violence, exploitation and abuse of children within the SDG Framework, Nueva York.

(2020d), "La violencia de género en situaciones de emergencia" [en línea] https://www.unicef.org/ spanish/protection/57929_58001.html.

_.(2020e), Para cada niño, reimaginemos un mundo mejor. Informe Anual de UNICEF 2019, Nueva York. 
_ (2019), Políticas favorables a la familia: rediseñar el lugar de trabajo del futuro [en línea] https://www. unicef.org/sites/default/files/2020-02/UNICEF-family-friendly-policies-es-2019.pdf.

_ (2018), Violencia en la primera infancia: una revisión teórica para América Latina y el Caribe. Resumen de políticas y recomendaciones de programa, Ciudad de Panamá.

_(2015), "Violence against Children" [en línea] https://data.unicef.org/topic/child-protection/violence/.

(2006), Behind Closed Doors: The Impact of Domestic Violence on Children, Nueva York.

UNICEF (Fondo de las Naciones Unidas para la Infancia)/Save the Children (2020), "Children in monetary poor households and COVID-19: Technical Note" [en línea] https://www.unicef.org/media/69656/file/ TechnicalNote-Children-living-in-monetary-poor-households-and-COVID-19.pdf.pdf.

UNODC (Oficina de las Naciones Unidas contra la Droga y el Delito) (2020), "Impact of the COVID-19 Pandemic on trafficking in persons", Thematic Brief, Viena.

Vaca Trigo, I. (2019), "Oportunidades y desafíos para la autonomía de las mujeres en el futuro escenario del trabajo", serie Asuntos de Género, No 54 (LC/TS.2019/3), Santiago, Comisión Económica para América Latina y el Caribe (CEPAL).

Este informe fue preparado por la Comisión Económica para América Latina y el Caribe (CEPAL) y la Oficina Regional para América Latina y el Caribe del Fondo de las Naciones Unidas para la Infancia (UNICEF). La Secretaria Ejecutiva de la CEPAL, Alicia Bárcena, el Director Regional Interino del UNICEF para América Latina y el Caribe, Bernt Aasen, y la Representante Especial del Secretario General sobre la Violencia contra los Niños, Najat Maalla M'jid, dirigieron su elaboración.

El informe fue elaborado por la División de Desarrollo Social de la CEPAL y las Unidades de Alianzas Públicas y de Protección de la Oficina Regional para América Latina y el Caribe del Fondo de las Naciones Unidas para la Infancia (UNICEF), con el apoyo del Consultor Fabio González.

Copyright (C) Naciones Unidas, 2020

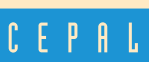

Comisión Económica para América Latina y el Caribe (CEPAL) www.cepal.org

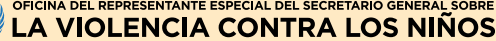

https://violenceagainstchildren.un.org/es

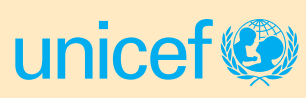

Oficina Regional para América Latina y el Caribe del Fondo de las Naciones Unidas para la Infancia (UNICEF) www.unicef.org/lac 